\title{
Prolonged Inner Retinal Photoreception Depends on the Visual Retinoid Cycle
}

\author{
저iwu Zhao, ${ }^{1}$ Weston Pack, ${ }^{1}$ Naheed W. Khan, ${ }^{1}$ and Kwoon Y. Wong ${ }^{1,2}$ \\ ${ }^{1}$ Department of Ophthalmology \& Visual Sciences and ${ }^{2}$ Department of Molecular, Cellular \& Developmental Biology, University of Michigan, Ann Arbor, \\ Michigan 48105
}

In addition to rods and cones, mammals have inner retinal photoreceptors called intrinsically photosensitive retinal ganglion cells (ipRGCs), which use the photopigment melanopsin and mediate nonimage-forming visual responses, such as pupil reflexes and circadian entrainment. After photic activation, photopigments must be reverted to their dark state to be light-sensitive again. For rods and to some extent cones, photopigment regeneration depends on the retinoid cycle in the adjacent retinal pigment epithelium (RPE). By contrast, ipRGCs are far from the RPE, and previous work suggests that melanopsin is capable of light-dependent self-regeneration. Here, we used in vitro ipRGC recording and in vivo pupillometry to show that the RPE is required for normal melanopsin-based responses to prolonged light, especially at high stimulus intensities. Melanopsin-based photoresponses of rat ipRGCs were remarkably sustained when a functional RPE was attached to the retina, but became far more transient if the RPE was removed, or if the retinoid cycle was inhibited, or when Müller glia were poisoned. Similarly, retinoid cycle inhibition markedly reduced the steady-state amplitude of melanopsin-driven pupil reflexes in both mice and rats. However, melanopsin photoresponses in RPE-separated rat retinas became more sustained in the presence of an 11-cis-retinal analog. In conclusion, during prolonged illumination, melanopsin regeneration depends partly on 11-cis-retinal from the RPE, possibly imported via Müller cells. Implications for RPE-related eye diseases and the acne drug isotretinoin (a retinoid cycle inhibitor) are discussed.

Key words: melanopsin; retina; RPE

\section{Significance Statement}

Intrinsically photosensitive retinal ganglion cells (ipRGCs) contain the photopigment melanopsin and drive subconscious physiological responses to light, e.g., pupillary constriction and neuroendocrine regulation. In darkness, each photopigment molecule in ipRGCs, as well as rod/cone photoreceptors, contains 11-cis-retinal (a vitamin A derivative) and light isomerizes it to all-transretinal, which activates the photopigment. To make this photopigment excitable again, all-trans-retinal must be reisomerized to 11-cis-retinal. For rods and to some extent cones, this reisomerization occurs in the adjacent retinal pigment epithelium (RPE), but because ipRGCs are far from the RPE, they are thought to regenerate excitable melanopsin exclusively through RPE-independent means. Here, we present electrophysiological and behavioral evidence that ipRGCs depend on the RPE to continuously regenerate melanopsin during intense prolonged photostimulation.

\section{Introduction}

The mammalian retina contains three classes of photoreceptors: rods, cones, and intrinsically photosensitive retinal ganglion cells

Received June 27, 2014; revised March 3, 2016; accepted March 8, 2016

Author contributions: K.Y.W. designed research; X.Z., W.P., N.W.K., and K.Y.W. performed research; X.Z. and K.Y.W. analyzed data; K.Y.W. wrote the paper.

This work was funded by National Eye Institute (NEI) Grants R00 EY018863 and R01 EY023660 to K.Y.W., a Research to Prevent Blindness Scientific Career Development Award to K.Y.W., and NEI Vision Core Grant P30 EY007003. We thank N. D. Perera for help with electroretinography.

The authors declare no competing financial interests.

Correspondence should be addressed to Kwoon Y. Wong, 1000 Wall Street, Ann Arbor, MI 48105. E-mail:kwoon@umich.edu.

DOI:10.1523/JNEUROSCI.2629-14.2016

Copyright $\odot 2016$ the authors $\quad 0270-6474 / 16 / 364209-09 \$ 15.00 / 0$
(ipRGCs). In a fully dark-adapted photoreceptor, its photopigment comprises an opsin apoprotein covalently linked to a light-absorbing chromophore, 11-cis-retinal (Walker et al., 2008). Light isomerizes 11-cis-retinal into all-trans-retinal, which activates the photopigment. To reset the pigment to its photoexcitable dark state, all-trans-retinal must be reisomerized to 11-cisretinal. For rods, reisomerization requires the retinoid cycle in the nearby retinal pigment epithelium (RPE), whereas for cones, it also involves the Müller glia's retinoid cycle (Wang and Kefalov, 2011). In contrast, because ipRGCs are located far from the $\mathrm{RPE}$, they have been proposed to regenerate their photopigment, melanopsin, independently of the RPE. Supporting this notion, the melanopsin-driven visual behaviors of rod/cone-degenerate mice were unaffected when the RPE retinoid cycle was abolished 

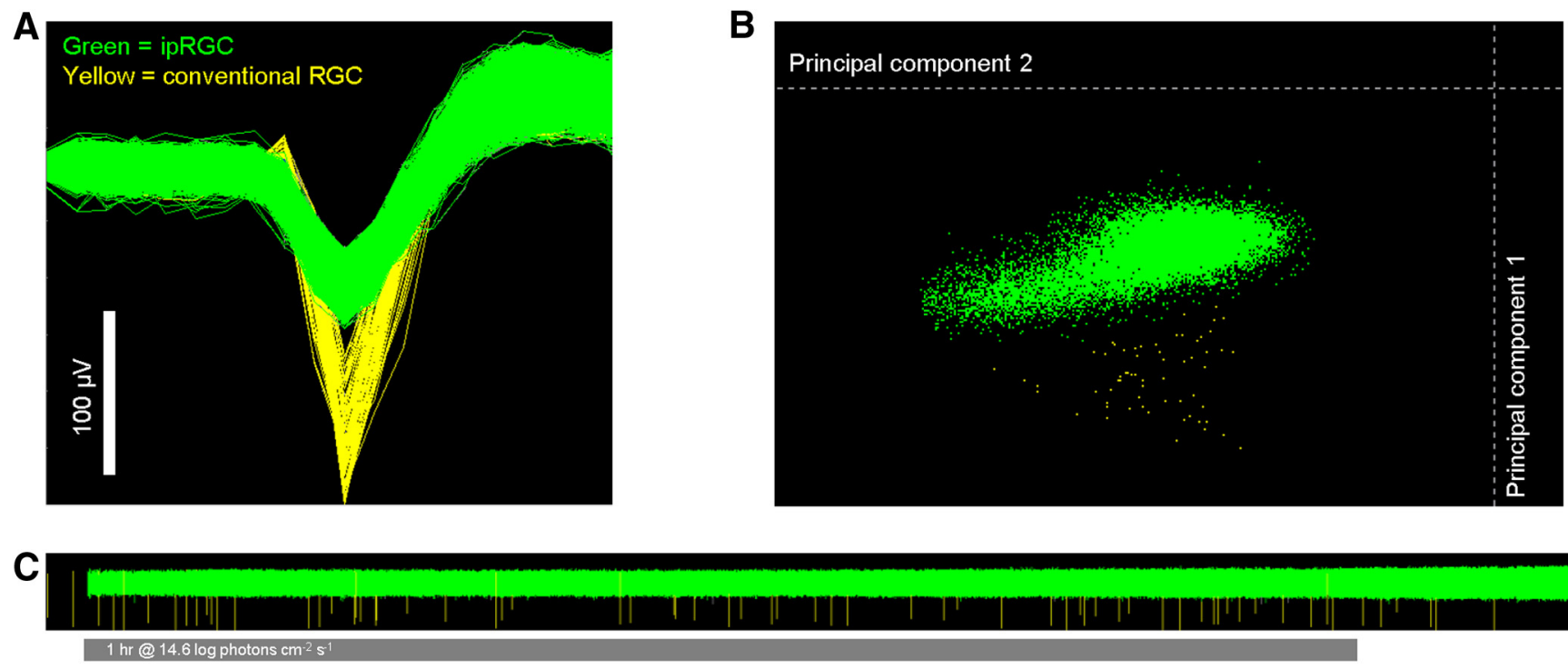

Figure 1. Cluster analysis of an MEA recording, which contains light-induced spikes from an ipRGC and spontaneous spikes from a conventional, nonphotoreceptive ganglion cell. This recording was made from an RPE-attached rat retina in the presence of control medium, and the stimulus was $1 \mathrm{~h} 480 \mathrm{~nm}$ light at $14.6 \log$ quanta $\mathrm{cm}^{-2} \mathrm{~s}^{-1}$. $\boldsymbol{A}$, Waveforms of the spikes from the ipRGC (green) and the other cell (yellow), which have significantly different shapes and amplitudes. $\boldsymbol{B}$, Principal component analysis of all spikes in this recording, with each dot representing one spike. The lateral dispersion of the ipRGC cluster (green) reflects the fact that spike amplitude increased gradually over the course of the 70 min recording. $C$, The entire recording, with the ipRGC spikes in green and the conventional ganglion cell's spikes in yellow.

(Doyle et al., 2006; Tu et al., 2006). Moreover, there is evidence that melanopsin can self-regenerate through photoreversal. That is, after photoexcitation, all-trans-retinal remains attached to melanopsin and is converted by subsequent light absorption to 11-cis-retinal (Fu et al., 2005; Koyanagi et al., 2005; Melyan et al., 2005; Panda et al., 2005; Mure et al., 2007, 2009; Davies et al., 2011) or another excitable state, 7-cisretinal (Matsuyama et al., 2012; Emanuel and Do, 2015).

However, melanopsin regeneration must also involve lightindependent mechanisms because previously light-adapted ipRGCs gradually regain intrinsic photosensitivity in darkness (Wong et al., 2005; Sexton et al., 2012b). Further, dark-adapted ipRGCs contain only 11-cis-retinal-bound melanopsin; if photoreversal was the sole regenerative mechanism, then a substantial amount of all-transretinal-bound molecules would also exist (Walker et al., 2008). The Müller-cell retinoid cycle is unlikely to contribute to melanopsin regeneration because ipRGCs seem unable to use cis-retinol (Sexton et al., 2012b), the retinoid synthesized by Müller cells for regenerating cone photopigments (Wang and Kefalov, 2011). These results led Sexton et al. to speculate that ipRGCs might possess a light-independent isomerase (Sexton et al., 2012a), although evidence remains lacking. In summary, the mechanisms that regenerate melanopsin are poorly understood. Here, we show that the RPE retinoid cycle is involved under certain photostimulation conditions.

\section{Materials and Methods}

Animals. All procedures were approved by the University Committee on Use and Care of Animals. The animals were 2-6-month-old Long-Evans rats and 5-10-month-old homozygous $r d 1$ mice, and both genders were used. Animals were maintained in a $12 \mathrm{~h}$ light/dark cycle and experiments were performed during the light phase. All animals were darkadapted overnight before each experiment.

Chemicals. The control medium was Ames' medium supplemented with $100 \mu \mathrm{M} \mathrm{L}-(+)$-2-amino-4-phosphonobutyric acid (L-AP4), $80 \mu \mathrm{M}$ 6,7-dinitroquinoxaline-2,3-dione (DNQX), $50 \mu \mathrm{M}$ D-(-)-2-amino-5phosphonopentanoic acid (D-AP5), $16 \mathrm{~mm}$ D-glucose, and $0.2-0.5 \%$ DMSO. DMSO was included in control medium because stock solutions of 9-cis-retinal, 13-cis-retinoic acid (13cisRA) and $\alpha$-phenyl- $N$-tertbutylnitrone (PBN) were DMSO-based. We purchased L-AP4, DNQX, and D-AP5 from Tocris Bioscience; 9-cis-retinol from Toronto Research Chemicals; xylazine, phenylephrine hydrochloride, and tropicamide from Akorn; proparacaine from Bausch \& Lomb; ketamine from Hospira; and all other chemicals from Sigma-Aldrich.

Multielectrode-array recording. Retinal preparations, light source, data acquisition, and spike analysis were as described previously (Wong, 2012). All retinas were superfused in darkness under each control or drug condition for $4-10 \mathrm{~h}$ before the $1 \mathrm{~h}$ light stimulation. ipRGC spikes were identified through thresholding and cluster analysis using Offline Sorter software (Plexon; Fig. 1). To calculate a cell's peak light-evoked spike rate, firing rate was averaged over $1 \mathrm{~s}$ bins and the highest rate identified, from which the mean rate of prestimulus spontaneous spiking (if any) was subtracted. To quantify the "sustainedness" of a light response, its "final-to-peak ratio" was calculated by dividing its average spike rate during the last $10 \mathrm{~min}$ of light by its peak spike rate. Statistical comparisons used the unpaired Student's $t$ test for normally distributed data and the Mann-Whitney $U$ test for non-normal data, with the significance level set at $p=0.05$. Error values are SEM.

Whole-cell recording. Whole-cell current-clamp recordings were obtained from $\alpha$-like, M4-type ipRGCs in Long-Evans rat eyecups, as described previously (Pack et al., 2015). We targeted this cell type because it can be identified relatively easily by its uniquely large soma (Reifler et al., 2015). Recordings were made after the eyecup had been incubated in $0.2 \%$ DMSO, $6 \mu \mathrm{M}$ 13cisRA, or $300 \mu \mathrm{M}$ PBN for 1-8 h. Ten-second depolarizing current steps were injected in $50 \mathrm{pA}$ increments, and the spikes evoked during the second half of the $150 \mathrm{pA}$ current step were used to calculate each cell's mean spike height and width. Spike height was measured from the point where membrane depolarization began to accelerate, and spike width was measured at half-height (see Fig. $3 E$, inset). The means from all cells were averaged to generate the plots shown in Figure $3 E$.

Electroretinography. Electroretinography (ERG) was performed using the Espion $\mathrm{E}^{2}$ system (Diagnosys). Rats were anesthetized with intraperitoneal injection of ketamine $(93 \mathrm{mg} / \mathrm{kg})$ and xylazine $(8 \mathrm{mg} / \mathrm{kg})$. Pupils were dilated with phenylephrine hydrochloride (2.5\%) and tropicamide $(1 \%)$. Body temperature was maintained at $37^{\circ} \mathrm{C}$ with a heating pad. After topical corneal anesthesia ( $0.5 \%$ proparacaine) and hydration $(2 \%$ methylcellulose), corneal ERGs were recorded using gold contact lens electrodes (Mayo). A gold wire loop put in the mouth served as reference, 
and the ground electrode was on the tail. Scotopic ERGs were evoked using $1 \mathrm{~ms}$ white flashes at $0.19 \log \mathrm{cd} \mathrm{s} / \mathrm{m}^{2}$.

Pupillometry. Before and throughout imaging, animals were anesthetized by ketamine ( $93 \mathrm{mg} / \mathrm{kg}$ for rats, $70 \mathrm{mg} / \mathrm{kg}$ for mice) and xylazine ( 8 $\mathrm{mg} / \mathrm{kg}$ ) and their body temperature maintained using a heating pad. We opted for anesthesia over head restraint due to the very prolonged recording duration, and because brain-state fluctuations in unanesthetized animals strongly influence pupil size (McGinley et al., 2015). DMSO (0.2 $\mathrm{ml}$ for rats and $0.02 \mathrm{ml}$ for mice) with or without 13 cisRA $(100 \mathrm{mg} / \mathrm{kg}$ ) was injected intraperitoneally. After the left eye's pupil had been dilated as described above, a syringe with a 30 gauge needle was used to puncture a hole through the ora serrata and inject intravitreally PBS (70 $\mu$ l for rats and $5 \mu \mathrm{l}$ for mice) containing $2 \mathrm{~mm}$ L-AP4, $2 \mathrm{~mm}$ DNQX, and $2 \mathrm{~mm}$ D-AP5. Following $1 \mathrm{~h}$ dark adaptation, a pupillometer (NeurOptics A-2000) was used to present $463 \mathrm{~nm}$ light onto the left eye and image the right eye's consensual pupil reflex. The final-to-peak ratio was calculated by dividing the average response amplitude during the last $2 \mathrm{~min}$ of photostimulation by the peak response. For rats, ERG was recorded from the left eye immediately after pupillometry to verify the drugs had blocked the b-wave.

Sodium iodate injection. While a rat was in a restrainer (Braintree Scientific), $0.4 \mathrm{ml}$ saline with sodium iodate $(50 \mathrm{mg} / \mathrm{kg}$ ) was injected into its tail vein. Animals were killed 4-6 d afterward for multielectrode array (MEA) recording.

\section{Results}

Normal melanopsin-based photoresponses require the RPE

In RPE-attached retinas superfused with Ames' medium containing rod/cone signaling blockers (control medium), ipRGCs generate very long-lasting melanopsin photoresponses (Wong, 2012). Figure $2 A$ shows averaged and representative responses to $1 \mathrm{~h} 480 \mathrm{~nm}$ light steps at $14.6 \log$ and $12.8 \log$ quanta $\mathrm{cm}^{-2} \mathrm{~s}^{-1}$ [for reference, a typical cloudy day is equivalent to $\sim 15.6 \mathrm{log}$ quanta $\mathrm{cm}^{-2} \mathrm{~s}^{-1}$ of $480 \mathrm{~nm}$ photons in terms of its ability to activate melanopsin (Enezi et al., 2011)]. Both responses peaked within $5 \mathrm{~min}$ and then decayed toward a steady state. In isolated, RPE-less retinas, melanopsin photoresponses also decayed after peaking, but the decay was faster and the steady-state amplitude was much lower (Fig. 2B). Final-to-peak response ratios were significantly lower than for RPE-attached retinas at both 14.6 and $12.8 \mathrm{log}$ intensities $(p<0.001)$. Peak spike rates were also lower than for RPE-attached retinas at $14.6 \log (33.0 \pm 1.8$ vs $43.9 \pm 4.3$ $\mathrm{Hz}, p=0.017)$, though statistically similar at $12.8 \log (p=$ $0.107)$. These differences could be due to the absence of the RPE, or due to mechanical damage inflicted during retinal isolation, or both. Thus, we next disrupted the RPE through intravenous injection of sodium iodate rather than by mechanical means. The melanopsin photoresponses in iodate-treated, RPE-attached retinas were still abnormally transient (Fig. 2C), with final-to-peak ratios being significantly lower than for untreated RPE-attached retinas ( $p<0.001$ at both intensities). However, though these retinas experienced less mechanical perturbation, peak spike rates were even lower $(15.4 \pm 1.2 \mathrm{~Hz}$ for $14.6 \mathrm{log}$ and $5.3 \pm 0.9 \mathrm{~Hz}$ for $12.8 \mathrm{log}$ ), probably due to iodate's toxic effect on retinal neurons (Tao et al., 2013).

\section{Importance of the RPE retinoid cycle}

Despite their shortcomings, the two previous experiments pointed to the possibility that a functional RPE is required for normal melanopsin photoresponses. The RPE's main functions are to maintain retinal health and to synthesize 11-cis-retinal through the retinoid cycle (Strauss, 1995), and so the abnormal light responses in Figure $2 B, C$ could be caused by poor retinal health or elimination of the retinoid cycle, or both. To test the hypothesis that these abnormal photoresponses were due to disruption of the retinoid cycle, we recorded from RPE-attached retinas in the presence of 3-10 $\mu \mathrm{M}$ 13cisRA or 200-500 $\mu \mathrm{M}$ PBN, which inhibit the RPE retinoid cycle (Sieving et al., 2001; Mandal et al., 2011). These drugs had similar effects and so the data were pooled. Compared with RPE-attached retinas in control medium, peak response amplitudes at both 14.6 and 12.8 log intensities were statistically similar $(p>0.05)$, but responses were abnormally transient (Fig. $2 D$ ) and had significantly lower finalto-peak ratios ( $p<0.001$ at both intensities). This result raised the possibility that 11-cis-retinal from the RPE helps ipRGCs regenerate melanopsin during prolonged illumination, thereby enabling them to keep responding at high spike rates. Two additional observations reinforced this possibility. First, while retinoid cycle inhibition made the responses to both intensities decay faster, it affected the higher-intensity response more: the $14.6 \mathrm{log}$ response in Figure $2 D$ became significantly smaller than that in Figure $2 A$ starting with the second $5 \mathrm{~min}$ bin, whereas the $12.8 \mathrm{log}$ responses from the two recording conditions did not differ significantly until the sixth bin (Fig. $2 D$, asterisks), suggesting that intense light exhausts melanopsin molecules faster and 11-cisretinal from the RPE is needed sooner to sustain the photoresponse. Second, in the presence of $100 \mu \mathrm{M}$ 9-cis-retinal (a commercially available analog of 11 -cis-retinal), the $14.6 \log$ photoresponses in isolated retinas became more tonic (Fig. 2E), with the final-to-peak ratio being significantly higher $(p=0.035)$ than that for the $14.6 \log$ response in Figure $2 B$ (isolated retinas in control medium), though it remained lower $(p<0.01)$ than the ratio for the $14.6 \log$ response in Figure $2 A$ (RPE-attached retinas in control medium), suggesting 9-cis-retinal reconstituted melanopsin only partially. The key final-to-peak ratios are shown in Figure $2 F$.

There was a potential caveat, however, because 13cisRA and/or PBN could have caused the above changes by acting on ipRGCs' spike-generation mechanisms rather than on the melanopsin photoresponse. This possibility was ruled out by two control experiments. First, when $6 \mathrm{mM} \mathrm{KCl}$ was added for $1 \mathrm{~h}$ to control medium containing $100 \mu \mathrm{M}$ picrotoxin, $5 \mu \mathrm{M}$ strychnine, and 13cisRA or PBN (picrotoxin and strychnine were included to block KCl-evoked amacrine-cell signaling), the firing rates of ipRGCs in isolated retinas were dramatically elevated throughout the hour (Fig. 3A), just as when the bath had DMSO instead of 13 cisRA or PBN (Fig. 3B). Incidentally, the Figure $3 B$ result further confirmed that the transient photoresponses detected in isolated retinas (Fig. $2 B$ ) were not caused by the retinal isolation procedure compromising ipRGCs' ability to generate prolonged spiking. In the second control, whole-cell recordings showed that M4 cells' spiking responses to current injection (Fig. 3C) were not significantly altered by these drugs, in terms of both firing rate (Fig. 3D) and spike waveform (Fig. 3C, bottom recordings, E). This result confirms that 13cisRA and PBN do not affect the voltage-gated channels underlying spike generation.

\section{Dependence on the retinoid cycle is behaviorally relevant}

We next examined the impact of retinoid cycle inhibition on the pupillary light reflex (PLR), a nonimage-forming visual behavior believed to be driven by both M1 and non-M1 ipRGCs (Ecker et al., 2010). To study melanopsin-mediated PLRs in Long-Evans rats, rod/cone signaling blockers were injected into the left eye whose pupil had been dilated. This treatment abolished the ERG b-wave, a field potential reflecting the light responses of bipolar cells (Fig. 4A), confirming effective block of rod/cone transmission to bipolar cells. Any residual signaling from bipolar cells to ganglion cells would probably be antagonized by DNQX and 

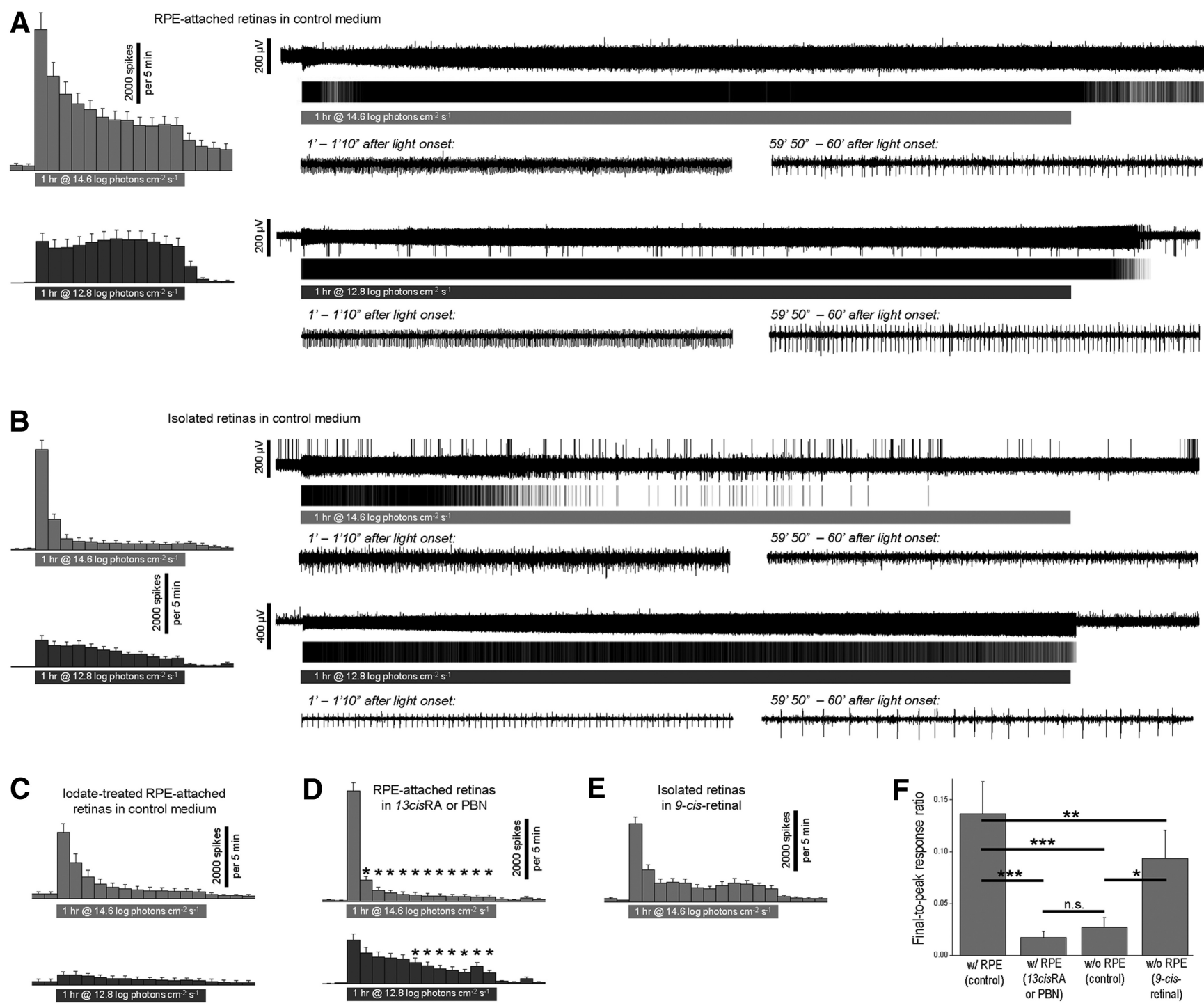

Figure 2. The generation of sustained melanopsin-based light responses requires the RPE retinoid cycle. $A$, Left, Averaged spike histograms illustrating the melanopsin-based photoresponses of ipRGCs in RPE-attached retinas, evoked by $480 \mathrm{~nm}$ light at $14.6 \log$ quanta $\mathrm{cm}^{-2} \mathrm{~s}^{-1}$ (top; $n=47$ cells) and $12.8 \log$ quanta $\mathrm{cm}^{-2} \mathrm{~s}^{-1}$ (bottom; $n=55$ cells). All error bars show SEM. Right, Two example recordings that contain relatively well isolated spikes from two different $\mathrm{ipRGCs}$, with the raster plots showing spike timings. The sporadic, nonlight-evoked vertical lines in these recordings include both spontaneous spikes from other, nonphotoreceptive ganglion cells and mechanical/electrical artifacts. To show individual spikes more clearly, two $10 \mathrm{~s}$ segments of each recording are displayed on an expanded time scale below the raster plot. $\boldsymbol{B}$, Melanopsin photoresponses of ipRGCs in isolated retinas. Left, Averaged spike histograms. $N=63$ cells for 14.6 log and 57 cells for 12.8 log. Right, Example recordings and raster plots. C, Melanopsin photoresponses of ipRGCs in RPE-attached retinas that had been treated with sodium iodate. $N=29$ cells for 14.6 log and 51 cells for $12.8 \log$. $D$, Melanopsin photoresponses recorded from RPE-attached retinas in the presence of retinoid cycle inhibitors. $N=48$ cells for $14.6 \log$ and 74 cells for $12.8 \log$. The asterisks mark the histogram columns that are significantly shorter $(p<0.05)$ than those shown in $\boldsymbol{A}$. $\boldsymbol{E}$, Melanopsin photoresponses recorded from isolated retinas in the presence of 9 -cis-retinal $(n=70$ cells). $\boldsymbol{F}$, A summary of the key results. These final-to-peak response ratios were calculated from the $14.6 \log$ responses shown in $\boldsymbol{A}, \boldsymbol{D}, \boldsymbol{B}$, and $\boldsymbol{E}$, respectively. ${ }^{*} p<0.05 ;{ }^{* *} p<0.01 ;{ }^{* * *} p<0.001 ; \mathrm{n}$. ., Not significantly different.

D-AP5. A $463 \mathrm{~nm}$ light with an intensity of $15.9 \log$ quanta $\mathrm{cm}^{-2}$ $\mathrm{s}^{-1}$ at the cornea was presented to the injected eye for $20 \mathrm{~min}$, and the other eye's consensual PLR imaged. PLRs were recorded both from control rats intraperitoneally injected with DMSO and from rats whose RPE retinoid cycle was inhibited by intraperitoneally injected 13cisRA (Sieving et al., 2001). Although all animals displayed PLRs lasting the duration of the stimulus, the 13cisRAinjected rats' responses decayed faster and final-to-peak response ratios were significantly smaller $(p=0.014$; Fig. $4 B)$. In contrast, the two animal groups' responses to a lower stimulus intensity (13.9 log quanta $\mathrm{cm}^{-2} \mathrm{~s}^{-1}$ ) had statistically indistinguishable final-to-peak ratios ( $p=0.457$; Fig. $4 C$ ), suggesting that retinoid cycle inhibition had a more pronounced impact at the higher light intensity.
Although the intraocularly injected drugs were expected to completely block rod/cone signaling to ganglion cells, this could not be known for certain. Thus, we also measured PLRs at the $15.9 \log$ intensity using aged homozygous $r d 1$ mice that were completely rodless and nearly coneless (Carter-Dawson et al., 1978). The residual cones do not appear sufficient to drive the PLR because knocking out melanopsin in $r d 1$ mice eliminates the PLR, as noted in a previous study (Panda et al., 2003), even though that study used younger (6-10 weeks) mice and did not include pharmacological blockade of rod/cone signaling. Nonetheless, to ensure that rod/cone transmission was completely absent, all our mice received intraocular injection of L-AP4, DNQX, and D-AP5. Compared with DMSO-injected control mice, the PLRs of the 13cisRA-injected mice had a significantly lower final- 
A Isolated retinas in 13cisRA or PBN + picrotoxin + strychnine
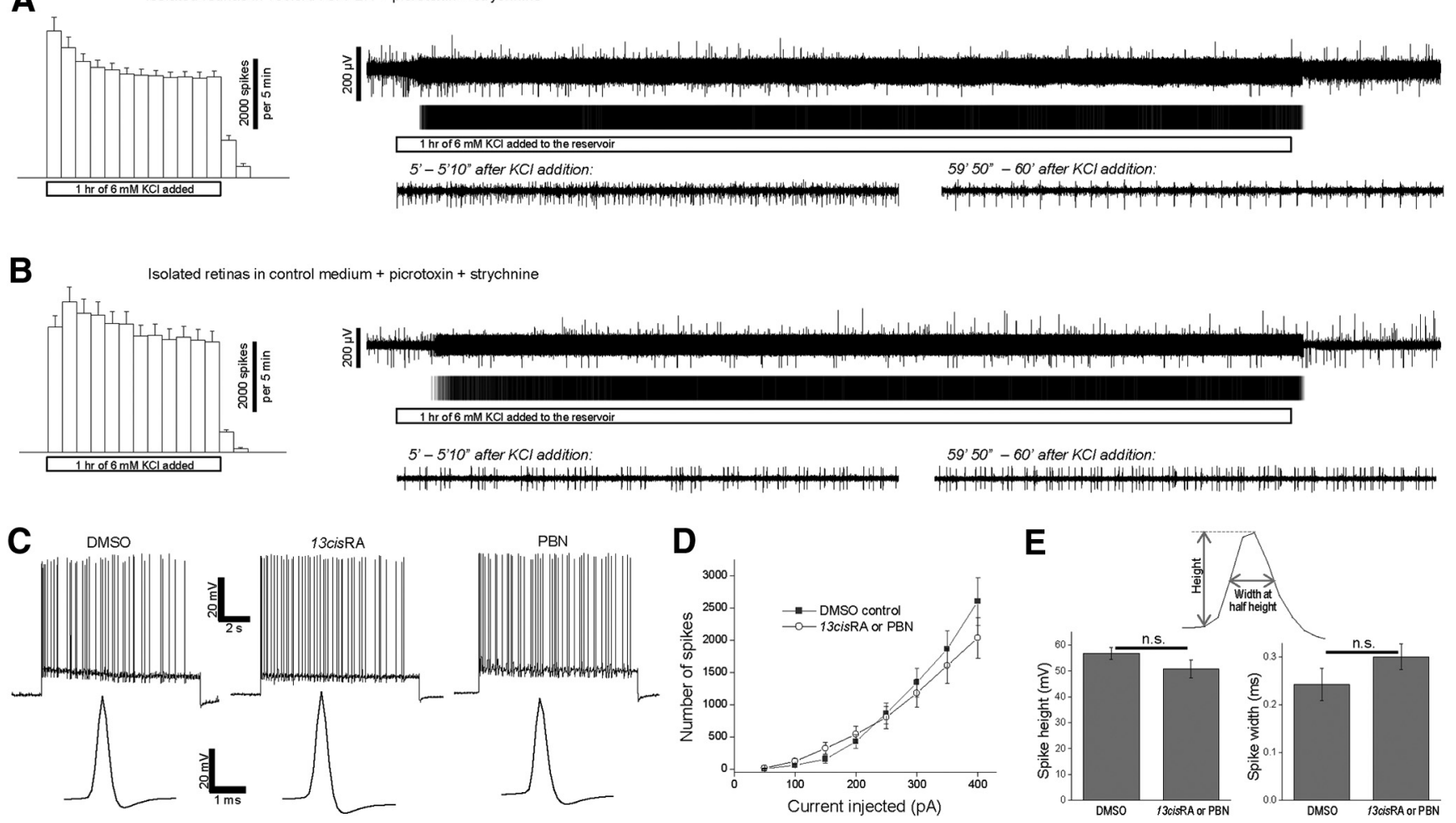

Figure 3. Control experiments revealed that the retinoid cycle inhibitors affected the melanopsin phototransduction cascade rather than ipRGCs' spiking mechanisms. $\boldsymbol{A}$, Adding 6 mm KCl to the reservoir increased ipRGC spiking for $1 \mathrm{~h}$ even when the superfusate contained 3-10 $\mu \mathrm{m}$ 13cisRA or $200 \mu \mathrm{m}$ PBN. Left, Averaged spike histogram generated from 36 ipRGCs in isolated retinas. Right, Example recording from one cell. $\boldsymbol{B}, 6 \mathrm{~mm}$ KCl induced a comparable spiking increase when the superfusate contained $0.2 \%$ DMSO instead of 13 cisRA or PBN. Left, Spike histogram averaged from 27 ipRGCs in isolated retinas. Right, Example recording from one cell. $\mathbf{C}-\boldsymbol{E}, 10$ s current steps of various amplitudes were applied to $11 \mathrm{M} 4$ cells in the presence of $0.3 \%$ DMSO, and to $16 \mathrm{M} 4$ cells in the presence of $10 \mu \mathrm{m}$ 13cisRA or $500 \mu \mathrm{m}$ PBN. C, Example $150 \mathrm{pA}$ responses (upper recordings) and individual spikes from these responses (bottom recordings). $D$, At each current amplitude, total spike counts for the two conditions were statistically similar ( $p>0.05$ ). $\boldsymbol{E}$, The spikes in the $150 \mathrm{pA}$ responses had statistically similar $(p>0.05)$ heights (left plot) and widths (right plot) in the two conditions. The inset illustrates how these two parameters were measured.

to-peak ratio ( $p=0.008$; Fig. $4 D$ ). The 13cisRA did not induce these effects by obstructing iris constriction or dilation because topically applied carbachol still fully constricted the pupils of 13cisRA-injected rats and mice (Fig. 4E), and because DMSOinjected and 13cisRA-injected animals had comparable darkadapted pupil sizes ( $p=0.45$ for rats and 0.24 for mice).

\section{Requirement for Müller glia}

Considering the great distance between the RPE and ipRGCs, an obvious question is how retinoids are transported between them. Since Müller cells span the thickness of the retina and interact closely with ipRGCs (Viney et al., 2007), we hypothesized that they transport 11-cis-retinal from the RPE to ipRGCs. To test whether these glia are required for ipRGCs to generate sustained intrinsic photoresponses, we obtained MEA recordings from RPE-attached retinas in the presence of 3-10 mM DL-2aminoadipic acid (DL-2-AAA), a Müller cell-specific gliotoxin (Pedersen and Karlsen, 1979). Compared with RPE-attached retinas in control medium (Fig. $2 A$ ), photoresponses recorded in the presence of DL-2-AAA were more transient and had lower finalto-peak ratios at both $14.6 \log (p<0.001)$ and $12.8 \log$ intensities $(p=0.017$; Fig. 5A). However, since DL-2-AAA is somewhat neurotoxic (Casper and Reif-Lehrer, 1983), it could have impaired ipRGCs' ability to spike tonically. We ruled out this possibility through a control experiment in which RPE-attached retinas were incubated in $100 \mu \mathrm{M} 9$-cis-retinal as well as DL-2AAA, which significantly increased the final-to-peak ratio $(p=$ 0.002; Fig. $5 B$ ), suggesting that the transient light responses in
Figure $5 A$ were not caused by an inability to spike tonically. This result also confirmed that those transient light responses were due to a loss of 11-cis-retinal.

While the requirement for Müller cells is consistent with the hypothesis that they traffic 11-cis-retinal from the RPE to ipRGCs, an alternative explanation is that ipRGCs use the 11-cisretinol made by these glia to regenerate excitable melanopsin, so that melanopsin regeneration was compromised when Müller cells were poisoned. This is unlikely the case, because the melanopsin photoresponses recorded from isolated retinas remained extremely transient in the presence of the 11-cis-retinol analog 9-cis-retinol (100 $\mu \mathrm{M}$; Fig. 5C), as previously reported for mouse ipRGCs (Sexton et al., 2012b). Reinforcing this conclusion, even though Müller cells presumably remained functional in isolated retinas superfused with control medium, the melanopsin photoresponses recorded from such retinas (Fig. $2 B$ ) were just as transient as those shown in Figure $5 A$.

\section{Responses to longer-wavelength light}

As mentioned (see Introduction), previous studies showed that melanopsin can regenerate itself through photoreversal, with the best wavelength for photoreversal being longer than the excitation $\lambda_{\max }$ of $480 \mathrm{~nm}$ (Koyanagi et al., 2005; Melyan et al., 2005; Mure et al., 2007, 2009; Matsuyama et al., 2012; Emanuel and Do, 2015). Thus, it is conceivable that in the absence of the retinoid cycle, the short-wavelength lights used in the above experiments (Figs. 2-5) were ineffective for reversing melanopsin and quickly exhausted excitable photopigments, resulting in transient 
A

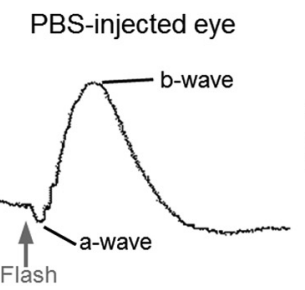

B1

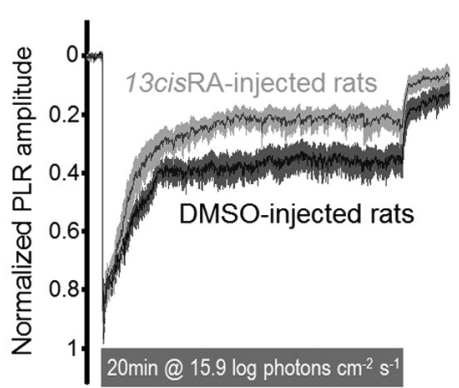

Eye injected with rod/cone signaling blockers
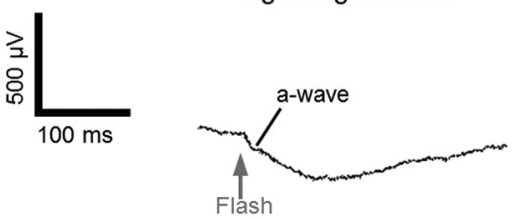

B2

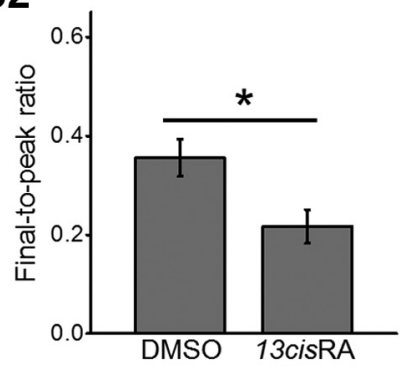

C2

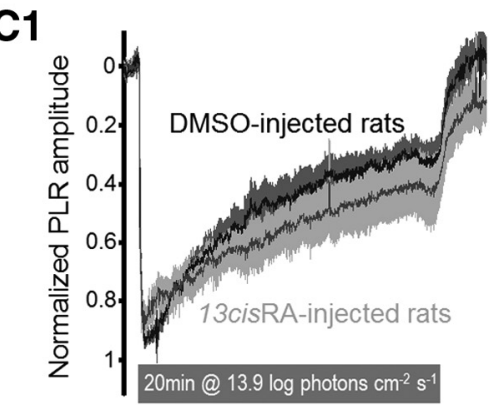

D1

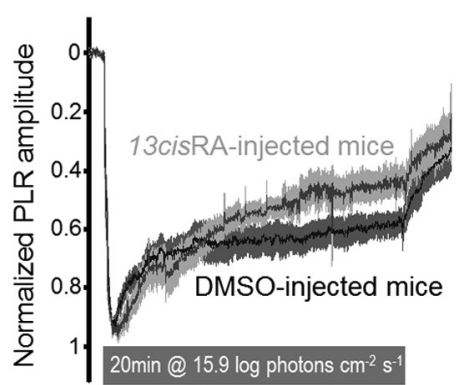

E1

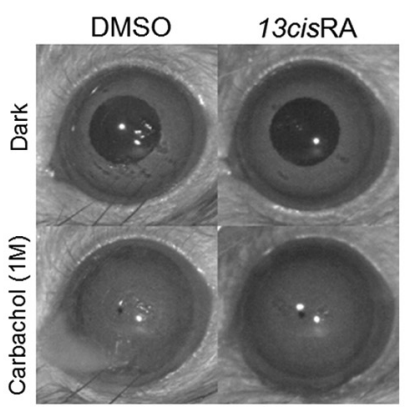

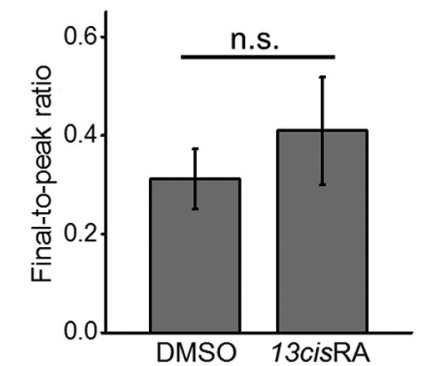

D2

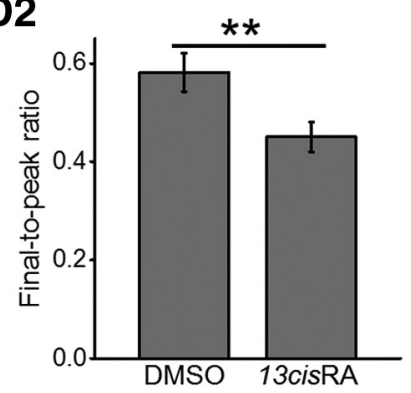

E2

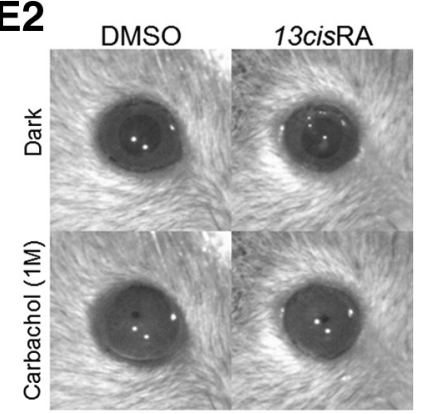

light responses. In the final experiment, we tested whether a longer-wavelength light, which is better for photoreversal, would evoke more sustained ipRGC responses in isolated retinas. Specifically, we tested $560 \mathrm{~nm}$ at $15.7 \mathrm{log}$ quanta $\mathrm{cm}^{-2} \mathrm{~s}^{-1}$, which should match the 14.6 $\log 480 \mathrm{~nm}$ stimulus in its ability to excite melanopsin (Berson et al., 2002). As a confirmation, these stimuli induced similar peak response amplitudes $(32.2 \pm 2.1 \mathrm{~Hz}$ for $15.7 \log 560 \mathrm{~nm}$ vs $33.0 \pm 1.8 \mathrm{~Hz}$ for $14.6 \log 480 \mathrm{~nm}, p=$ 0.284 ). But since the peak wavelength for photoreversal is red-shifted, this 560 $\mathrm{nm}$ light should be better than the 480 $\mathrm{nm}$ stimulus for photoreversing melanopsin. Though the averaged $560 \mathrm{~nm}$ response seemed slightly more sustained (compare Figs. 2B, 6), the finalto-peak ratios were not significantly different $(p=0.401)$.

\section{Discussion}

Reconciling with previous studies

Three prior studies that examined ipRGCs' reliance on the RPE by either knocking out critical components of the retinoid cycle (rpe65 and lrat) or inhibiting this cycle pharmacologically gave divergent results. Fu et al. (2005) showed that, with rpe65 knocked out, the melanopsin-based photosensitivity of both the PLR and ipRGCs in 1-3month-old gnat $1^{-1-} \mathrm{cnga}^{-1-}$ mice (which have physically intact but nonfunctional rods and cones) was reduced up to 40-fold. In contrast, Doyle et al. (2006) reported that 2-3-month-old rpe65 ${ }^{-1-}$ rdta mice (which are rodless) had normal circadian phaseshift responses to a $15 \mathrm{~min}, \sim 11 \log$ quanta $\mathrm{cm}^{-2} \mathrm{~s}^{-1}$ light pulse. Tu et al. (2006) tested $30 \mathrm{~s}$ lights of various intensities and found that while knocking out rpe65 or lrat in 7-8month-old $r d t a$ or $r d 1$ mice reduced PLR sensitivity 20 -fold, acute pharmacologic inhibition of the retinoid cycle in $r d t a$ or $r d 1$ mice had no impact. These findings led Tu et al. to propose that melanopsin photosensitivity does not acutely require the RPE because ipRGCs have an intrinsic capacity to regenerate melanopsin using both lightdependent and light-independent means, although it is impaired by chronic retinoid depletion such as occurs in $r p e 65^{-1-}$ ani-

\section{$\leftarrow$}

Figure 4. Inhibition of the retinoid cycle made the melanopsin-driven PLR less sustained. In this experiment, rod/cone signaling blockers were injected into the eyes of both Long-Evans rats and homozygous $r d 1$ mice. $A$, To verify that the injected drugs were effective on the rats, ERGs were recorded from a sham-injected eye (left) and an eye injected with rod/cone signaling blockers (right). The lack of a b-wave (right) confirmed that rod/cone signaling to bipolar cells was blocked. B1, Averaged PLRs of rats intraperitoneally injected with 13cisRA (gray trace) versus DMS0-injected rats (dark trace), with peak amplitude normalized to 1. The shaded areas around the traces show SEM. The stimulus was a $463 \mathrm{~nm}$ light at $15.9 \log$ quanta $\mathrm{cm}^{-2} \mathrm{~s}^{-1} . N=11$ rats for the DMSO injection and 8 rats for 13cisRA. B2, 13cisRA reduced the final-to-peak amplitude ratio. , When stimulus intensity was reduced to $13.9 \log$ quanta $\mathrm{cm}^{-2} \mathrm{~s}^{-1}, 13$ cisRA did not significantly alter the final-to-peak response ratio. $N=8$ rats for DMSO and

6 rats for 13cisRA. C1, Averaged PLRs. C2, Averaged final-topeak amplitude ratios. $\boldsymbol{D}$, The final-to-peak response ratios of rd1 mice were significantly reduced by 13 cisRA. $N=9$ mice for DMS0 and 8 mice for 13cisRA. D1, Averaged PLRs. D2, Averaged final-to-peak ratios. $\boldsymbol{E}$, Topically applied $1 \mathrm{~m}$ carbachol fully constricted the pupils of both DMSO-injected and 13cisRA-injected rats (E1) and rd1 mice (E2) within $5 \mathrm{~min}$. 

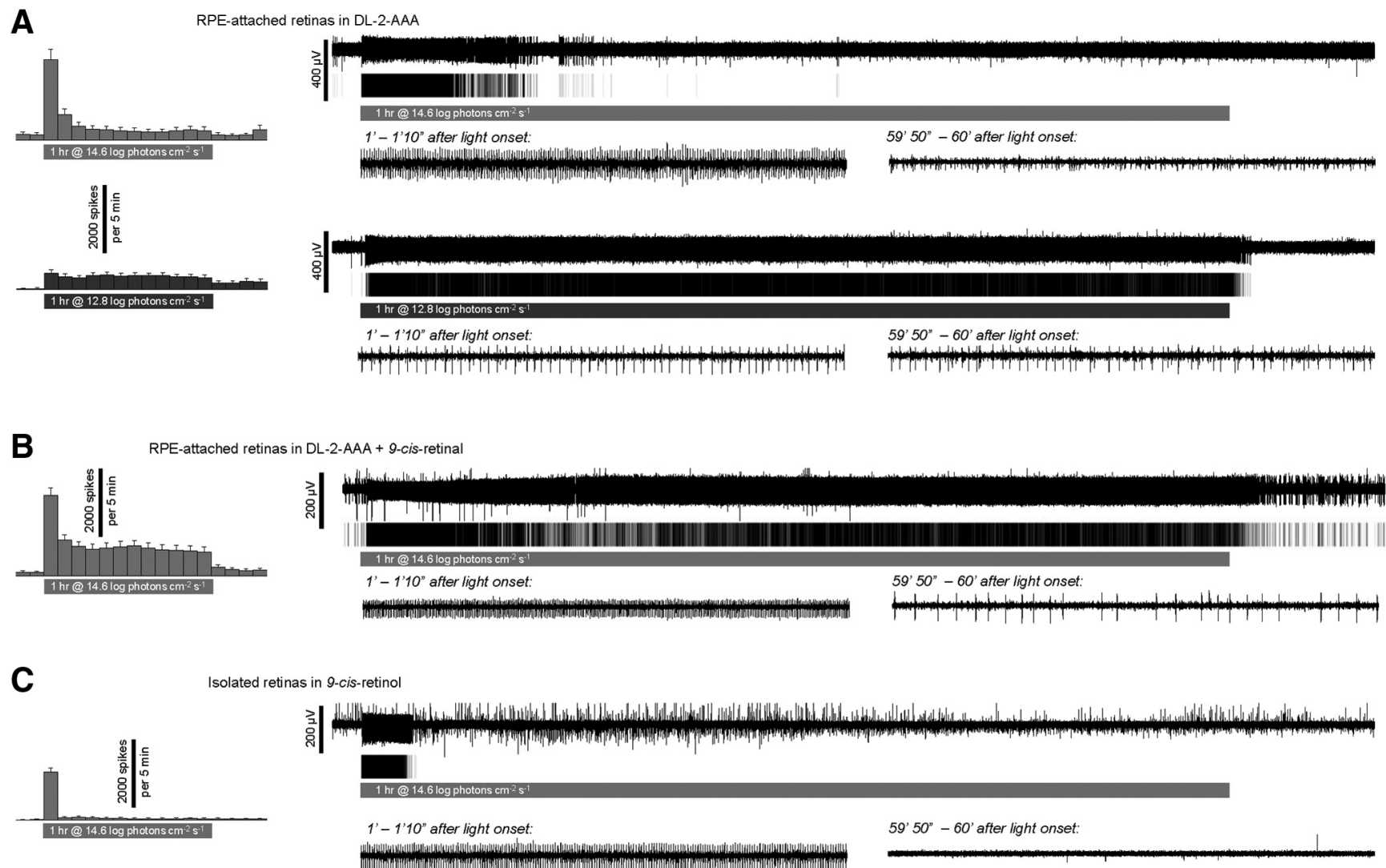

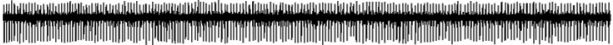

Figure 5. Poisoning the Müller cells in RPE-attached retinas made melanopsin photoresponses more transient. $A$, Melanopsin photoresponses recorded from RPE-attached retinas in the presence of the gliotoxin DL-2-AAA. Left, Averaged spike histograms. $N=35$ cells for $14.6 \mathrm{log}$ and 46 cells for $12.8 \mathrm{log}$. Right, Example recordings and raster plots. $B$, A control confirming that the transient light responses recorded in the presence of DL-2-AAA ( $\boldsymbol{A})$ were due to a loss in 11-cis-retinal, and that this gliotoxin did not prevent ipRGCs from spiking tonically. In this experiment, RPE-attached retinas were superfused with control medium containing $100 \mu \mathrm{m}$ 9-cis-retinal as well as $3 \mu \mathrm{m}$ DL-2-AAA. Left, Averaged spike histogram. $N=41$ cells. Right, An example recording. C, $100 \mu \mathrm{m}$ 9-cis-retinol failed to make the intrinsic photoresponses of ipRGCs in isolated retinas more sustained. Left, Averaged spike histogram. $N=56$ cells. Right, An example recording.

mals. To explain why rodless $r p e 65^{-/-}$mice gave stronger melanopsin-driven photoresponses than $r p e 65^{-/-}$mice with intact photoreceptors, rods were speculated to have an inhibitory influence on ipRGCs, possibly by acting as a chromophore sink (Doyle et al., 2006; Tu et al., 2006).

Here, we tested longer and higher-intensity stimuli and found that acutely inhibiting the RPE retinoid cycle in wild-type retinas reduced melanopsin photosensitivity in a time-dependent and intensity-dependent way: during continuous stimulation by intense light $\left(\sim 15 \log\right.$ quanta $\left.\mathrm{cm}^{-2} \mathrm{~s}^{-1}\right)$, sensitivity was attenuated after just several minutes, whereas at $\sim 13 \log$ quanta $\mathrm{cm}^{-2} \mathrm{~s}^{-1}$, it was not significantly affected for much longer. Even in homozygous $r d 1$ mice, where rods' presumed inhibitory effect on ipRGCs is eliminated and ipRGCs are physically closer to the RPE, normal melanopsin-driven PLR responses to prolonged intense light still required the RPE retinoid cycle. To reconcile our results with the three earlier studies, we propose the following. ipRGCs' intrinsic mechanisms for pigment regeneration can sustain responses to low light intensities for long periods, and may also help melanopsin dark-adapt after light adaptation (Wong et al., 2005). But during intense illumination, these intrinsic mechanisms cannot keep up with the high demand for excitable photopigments, and 11-cis-retinal from the RPE serves to speed up melanopsin reformation and maintain a robust photoresponse. Thus, at high stimulus intensities, as little as several minutes of RPE retinoid depletion can begin to impair melanopsin photosensitivity. This model can also explain the effects of age and genetic background observed in the three prior studies. All animals were housed in

Isolated retinas in control medium; $\underline{560 \mathrm{~nm}}$ at $15.7 \log$ photons $\mathrm{cm}^{-2} \mathrm{~s}^{-1}$
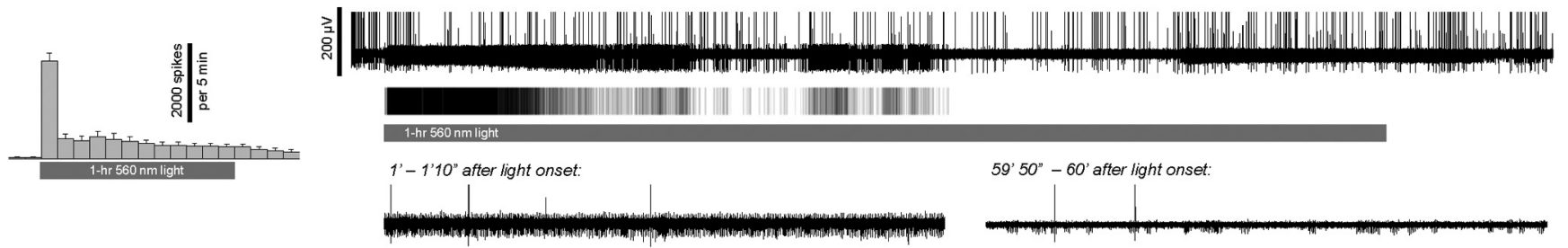

Figure 6. The dependence on the RPE extends to longer wavelengths. Melanopsin photoresponses were recorded from isolated retinas, using a $560 \mathrm{~nm}$ stimulus that was as effective for activating melanopsin as the $14.6 \mathrm{log} 480 \mathrm{~nm}$ light used in the other experiments, but should be more effective for melanopsin photoreversal. Left, Averaged spike histogram. $N=61$ cells. Right, An example recording. 
daily light/dark cycles. Each day, melanopsin in the $r p e 65^{-/-}$and rat $^{-1-}$ animals was bleached substantially during the light phase and then restored in the dark phase, but probably incompletely. Thus, whereas melanopsin photoreception still appeared normal in 2-3-month-old rpe65 $5^{-1-} r d t a$ mice (Doyle et al., 2006), it was reduced 20-fold 5 months later (Tu et al., 2006). In contrast, 1-3-month-old rpe65 $5^{-/-}$gnat $1^{-/-}$cnga $^{-/-}$mice already showed significant deficits because their rods and cones competed with ipRGCs for the low level of 9-cis-retinal available (Fan et al., 2003), thereby accelerating melanopsin depletion.

\section{An unexpected role for the RPE retinoid cycle}

There are three reasons why ipRGCs' dependence on the RPE retinoid cycle is surprising. First, melanopsin can regenerate selfsufficiently via photoreversal because its all-trans-retinal-bound excited state can be reverted to the 11-cis-retinal-bound dark state through photon absorption. But, as explained (see Introduction), photoreversal cannot be the only mechanism for regenerating melanopsin. Moreover, even invertebrate photopigments well known to be bistable and capable of photoreversal undergo slow regeneration in the absence of light (Hillman et al., 1983). In addition, Montell and colleagues showed that the maintenance of Drosophila rhodopsin depends on the retinoid cycle in retinal pigment cells, which are equivalent to the mammalian RPE, suggesting that "a bistable pigment and a visual cycle are not exclusive" and that "mammalian photoreceptor cells that use bistable rhodopsin-like receptors also depend on enzymatic visual cycles" (Wang et al., 2010). In fact, during prolonged exposure to intense light, melanopsin reformation may depend more on the RPE than on photoreversal, even for a long-wavelength stimulus thought to be potent for photoreversal (compare Figs. 2A, 6). We further noticed that the response to such a stimulus was statistically indistinguishable from that induced by a shorter wavelength (Fig. 2B, top), mirroring Lucas and colleagues' finding that the behavioral responses of rodless, coneless mice to broad-spectrum lights could be predicted simply by weighting these spectra according to the nomogram for melanopsin excitation (Enezi et al., 2011).

Melanopsin photoreversal has led to the view that photoactivation should not release all-trans-retinal from the opsin apoprotein, and this is the second reason why the RPE's contribution to melanopsin reformation is surprising: such contribution requires all-trans-retinal to dissociate from melanopsin so that it can be replaced with exogenous 11-cis-retinal. Indeed, Sexton et al. found melanopsin to be highly resistant to bleaching by hydroxylamine or bright light that could easily release 11-cis-retinal from rod/cone photopigments (Sexton et al., 2012b). But hydroxylamine does bleach melanopsin somewhat (Walker et al., 2008; Matsuyama et al., 2012), and 1 h of illumination by white fluorescent light equivalent to $\sim 14.7 \log$ quanta $\mathrm{cm}^{-2} \mathrm{~s}^{-1}$ of $480 \mathrm{~nm}$ light (Enezi et al., 2011) strongly bleaches melanopsin (Jones et al., 2013). Further, adding 9-cis-retinal to light-adapted ipRGCs increased melanopsin photosensitivity (Fu et al., 2005; Do et al., 2009). Yau and colleagues cautioned that ipRGCs might have bare melanopsin molecules that are normally unused, which could combine with the applied 9-cis-retinal to form functional photopigments. However, we found that adding 9-cis-retinal to dark-adapted ipRGCs did not increase their peak spike rates (Fig. 2 , compare $B, E$ ), indicating that dark-adapted ipRGCs contain few if any bare melanopsin molecules. In summary, there is ample evidence that melanopsin is bleachable, making it possible for exogenous 11-cis-retinal to reconstitute photopigments. Never- theless, melanopsin does appear to retain the chromophore more tightly than rod/cone photopigments (Thompson et al., 2001; Sexton et al., 2012b). Thus, it is reasonable to hypothesize that while melanopsin is less bleachable than rod/cone photopigments, it can be bleached to some extent, thereby allowing exogenous 11-cis-retinal to contribute to pigment reformation.

Finally, the physical distance separating ipRGCs from the RPE makes their interaction surprising. We hypothesized that Müller cells traffic retinoids from the RPE to ipRGCs because they are the only cells that span the retina and because they associate intimately with ipRGCs (Viney et al., 2007), and our DL-2-AAA data support this hypothesis (Fig. 5A). Cellular retinaldehyde-binding protein (CRALBP) is distributed throughout Müller cells, with the highest density near ganglion cells (Bunt-Milam and Saari, 1983). While CRALBP in the outer retina facilitates cones' dark adaptation (Xue et al., 2015), its role in the inner retina remains mysterious. We propose that it shuttles retinoids from the RPE to ipRGCs. Although CRALBP does not appear necessary for mice to generate normal melanopsin-driven PLR responses to $30 \mathrm{~s}$ light (Xue et al., 2015), its requirement for responses to much longer photostimulation has not been examined. Müller cells could conceivably also contribute directly to melanopsin regeneration. Even though Müller cells do not synthesize 11-cis-retinal (Wang and Kefalov, 2011) and the 11-cis-retinol they produce cannot be used by ipRGCs, it remains possible that they synthesize an unidentified retinoid that ipRGCs can use. However, even if such a mechanism exists, it is unlikely to make a dominant contribution during prolonged illumination because the light responses recorded from isolated retinas (where this mechanism would be functional) were not noticeably more tonic than those recorded in the presence of DL-2-AAA (compare Figs. 2B, 5A).

\section{Clinical relevance}

The retinoid cycle inhibitor 13cisRA is also called isotretinoin and is used as a treatment for severe acne. Due to its inhibition of the RPE retinoid cycle, isotretinoin causes poor night vision in some patients (Weleber et al., 1986; Sieving et al., 2001). Our data suggest that it could also attenuate melanopsin-based photoreception and thus compromise ipRGC-driven photoresponses, such as circadian entrainment, mood enhancement, and sleep regulation, a hypothesis first proposed by Szabo (2007). This action on ipRGCs could contribute to some of the psychiatric side effects of this drug (Kontaxakis et al., 2009).

Our findings further suggest that ipRGC function may be impaired in eye diseases involving disruptions of the RPE retinoid cycle, such as Leber's congenital amaurosis (Gu et al., 1997; Marlhens et al., 1997). Considering that retinoid cycle inhibition caused melanopsin responses to prolonged intense light to become more transient than those evoked by lower intensities (Fig. $2 D$ ), moderate ambient light levels may be the best for promoting nonimage-forming photoreception in these patients.

\section{References}

Berson DM, Dunn FA, Takao M (2002) Phototransduction by retinal ganglion cells that set the circadian clock. Science 295:1070-1073. CrossRef Medline

Bunt-Milam AH, Saari JC (1983) Immunocytochemical localization of two retinoid-binding proteins in vertebrate retina. J Cell Biol 97:703-712. CrossRef Medline

Carter-Dawson LD, LaVail MM, Sidman RL (1978) Differential effect of the rd mutation on rods and cones in the mouse retina. Invest Ophthalmol Vis Sci 17:489-498. Medline

Casper DS, Reif-Lehrer L (1983) Effects of alpha-aminoadipate isomers on the morphology of the isolated chick embryo retina. Invest Ophthalmol Vis Sci 24:1480-1488. Medline 
Davies WI, Zheng L, Hughes S, Tamai TK, Turton M, Halford S, Foster RG, Whitmore D, Hankins MW (2011) Functional diversity of melanopsins and their global expression in the teleost retina. Cell Mol Life Sci 68: 4115-4132. CrossRef Medline

Do MT, Kang SH, Xue T, Zhong H, Liao HW, Bergles DE, Yau KW (2009) Photon capture and signalling by melanopsin retinal ganglion cells. Nature 457:281-287. CrossRef Medline

Doyle SE, Castrucci AM, McCall M, Provencio I, Menaker M (2006) Nonvisual light responses in the Rpe65 knockout mouse: rod loss restores sensitivity to the melanopsin system. Proc Natl Acad Sci U S A 103: 10432-10437. CrossRef Medline

Ecker JL, Dumitrescu ON, Wong KY, Alam NM, Chen SK, LeGates T, Renna JM, Prusky GT, Berson DM, Hattar S (2010) Melanopsin-expressing retinal ganglion-cell photoreceptors: cellular diversity and role in pattern vision. Neuron 67:49-60. CrossRef Medline

Emanuel AJ, Do MT (2015) Melanopsin tristability for sustained and broadband phototransduction. Neuron 85:1043-1055. CrossRef Medline

Enezi Ja, Revell V, Brown T, Wynne J, Schlangen L, Lucas R (2011) A "melanopic" spectral efficiency function predicts the sensitivity of melanopsin photoreceptors to polychromatic lights. J Biol Rhythms 26:314-323. CrossRef Medline

Fan J, Rohrer B, Moiseyev G, Ma JX, Crouch RK (2003) Isorhodopsin rather than rhodopsin mediates rod function in RPE65 knock-out mice. Proc Natl Acad Sci U S A 100:13662-13667. CrossRef Medline

Fu Y, Zhong H, Wang MH, Luo DG, Liao HW, Maeda H, Hattar S, Frishman LJ, Yau KW (2005) Intrinsically photosensitive retinal ganglion cells detect light with a vitamin A-based photopigment, melanopsin. Proc Natl Acad Sci U S A 102:10339-10344. CrossRef Medline

Gu SM, Thompson DA, Srikumari CR, Lorenz B, Finckh U, Nicoletti A, Murthy KR, Rathmann M, Kumaramanickavel G, Denton MJ, Gal A (1997) Mutations in RPE65 cause autosomal recessive childhood-onset severe retinal dystrophy. Nat Genet 17:194-197. CrossRef Medline

Hillman P, Hochstein S, Minke B (1983) Transduction in invertebrate photoreceptors: role of pigment bistability. Physiol Rev 63:668-772. Medline

Jones KA, Hatori M, Mure LS, Bramley JR, Artymyshyn R, Hong SP, Marzabadi M, Zhong H, Sprouse J, Zhu Q, Hartwick AT, Sollars PJ, Pickard GE, Panda S (2013) Small-molecule antagonists of melanopsin-mediated phototransduction. Nat Chem Biol 9:630-635. CrossRef Medline

Kontaxakis VP, Skourides D, Ferentinos P, Havaki-Kontaxaki BJ, Papadimitriou GN (2009) Isotretinoin and psychopathology: a review. Ann Gen Psychiatry 8:2. CrossRef Medline

Koyanagi M, Kubokawa K, Tsukamoto H, Shichida Y, Terakita A (2005) Cephalochordate melanopsin: evolutionary linkage between invertebrate visual cells and vertebrate photosensitive retinal ganglion cells. Curr Biol 15:1065-1069. CrossRef Medline

Mandal MN, Moiseyev GP, Elliott MH, Kasus-Jacobi A, Li X, Chen H, Zheng L, Nikolaeva O, Floyd RA, Ma JX, Anderson RE (2011) Alpha-phenyl$\mathrm{N}$-tert-butylnitrone (PBN) prevents light-induced degeneration of the retina by inhibiting RPE65 protein isomerohydrolase activity. J Biol Chem 286:32491-32501. CrossRef Medline

Marlhens F, Bareil C, Griffoin JM, Zrenner E, Amalric P, Eliaou C, Liu SY, Harris E, Redmond TM, Arnaud B, Claustres M, Hamel CP (1997) Mutations in RPE65 cause Leber's congenital amaurosis. Nat Genet 17: 139-141. CrossRef Medline

Matsuyama T, Yamashita T, Imamoto Y, Shichida Y (2012) Photochemical properties of mammalian melanopsin. Biochemistry 51:5454-5462. CrossRef Medline

McGinley MJ, Vinck M, Reimer J, Batista-Brito R, Zagha E, Cadwell CR, Tolias AS, Cardin JA, McCormick DA (2015) Waking state: rapid variations modulate neural and behavioral responses. Neuron 87:1143-1161. CrossRef Medline

Melyan Z, Tarttelin EE, Bellingham J, Lucas RJ, Hankins MW (2005) Addition of human melanopsin renders mammalian cells photoresponsive. Nature 433:741-745. CrossRef Medline

Mure LS, Rieux C, Hattar S, Cooper HM (2007) Melanopsin-dependent nonvisual responses: evidence for photopigment bistability in vivo. J Biol Rhythms 22:411-424. CrossRef Medline

Mure LS, Cornut PL, Rieux C, Drouyer E, Denis P, Gronfier C, Cooper HM (2009) Melanopsin bistability: a fly's eye technology in the human retina. PLoS One 4:e5991. CrossRef Medline
Pack W, Hill DD, Wong KY (2015) Melatonin modulates M4-type ganglion-cell photoreceptors. Neuroscience 303:178-188. CrossRef Medline

Panda S, Provencio I, Tu DC, Pires SS, Rollag MD, Castrucci AM, Pletcher MT, Sato TK, Wiltshire T, Andahazy M, Kay SA, Van Gelder RN, Hogenesch JB (2003) Melanopsin is required for non-image-forming photic responses in blind mice. Science 301:525-527. CrossRef Medline

Panda S, Nayak SK, Campo B, Walker JR, Hogenesch JB, Jegla T (2005) Illumination of the melanopsin signaling pathway. Science 307:600-604. CrossRef Medline

Pedersen OO, Karlsen RL (1979) Destruction of Muller cells in the adult rat by intravitreal injection of D, L-alpha-aminoadipic acid. An electron microscopic study. Exp Eye Res 28:569-575. CrossRef Medline

Reifler AN, Chervenak AP, Dolikian ME, Benenati BA, Meyers BS, Demertzis ZD, Lynch AM, Li BY, Wachter RD, Abufarha FS, Dulka EA, Pack W, Zhao X, Wong KY (2015) The rat retina has five types of ganglion-cell photoreceptors. Exp Eye Res 130:17-28. CrossRef Medline

Sexton T, Buhr E, Van Gelder RN (2012a) Melanopsin and mechanisms of non-visual ocular photoreception. J Biol Chem 287:1649-1656. CrossRef Medline

Sexton TJ, Golczak M, Palczewski K, Van Gelder RN (2012b) Melanopsin is highly resistant to light and chemical bleaching in vivo. J Biol Chem 287:20888-20897. CrossRef Medline

Sieving PA, Chaudhry P, Kondo M, Provenzano M, Wu D, Carlson TJ, Bush RA, Thompson DA (2001) Inhibition of the visual cycle in vivo by 13-cis retinoic acid protects from light damage and provides a mechanism for night blindness in isotretinoin therapy. Proc Natl Acad Sci U S A 98: 1835-1840. CrossRef Medline

Strauss O (1995) The retinal pigment epithelium. In: Webvision: the organization of the retina and visual system (Kolb H, Fernandez E, Nelson R, eds). Salt Lake City, UT: University of Utah Health Sciences Center.

Szabo B (2007) Antiandrogenic effect of isotretinoin: is the retina involved in mechanism of action? Med Hypotheses 69:1281-1283. CrossRef Medline

Tao Z, Dai J, He J, Li C, Li Y, Yin ZQ (2013) The influence of NaIO(3)induced retinal degeneration on intra-retinal layer and the changes of expression profile/morphology of DA-ACs and mRGCS. Mol Neurobiol 47:241-260. CrossRef Medline

Thompson CL, Blaner WS, Van Gelder RN, Lai K, Quadro L, Colantuoni V, Gottesman ME, Sancar A (2001) Preservation of light signaling to the suprachiasmatic nucleus in vitamin A-deficient mice. Proc Natl Acad Sci U S A 98:11708-11713. CrossRef Medline

Tu DC, Owens LA, Anderson L, Golczak M, Doyle SE, McCall M, Menaker M, Palczewski K, Van Gelder RN (2006) Inner retinal photoreception independent of the visual retinoid cycle. Proc Natl Acad Sci U S A 103:1042610431. CrossRef Medline

Viney TJ, Balint K, Hillier D, Siegert S, Boldogkoi Z, Enquist LW, Meister M, Cepko CL, Roska B (2007) Local retinal circuits of melanopsincontaining ganglion cells identified by transsynaptic viral tracing. Curr Biol 17:981-988. CrossRef Medline

Walker MT, Brown RL, Cronin TW, Robinson PR (2008) Photochemistry of retinal chromophore in mouse melanopsin. Proc Natl Acad Sci U S A 105:8861-8865. CrossRef Medline

Wang JS, Kefalov VJ (2011) The cone-specific visual cycle. Prog Retin Eye Res 30:115-128. CrossRef Medline

Wang X, Wang T, Jiao Y, von Lintig J, Montell C (2010) Requirement for an enzymatic visual cycle in Drosophila. Curr Biol 20:93-102. CrossRef Medline

Weleber RG, Denman ST, Hanifin JM, Cunningham WJ (1986) Abnormal retinal function associated with isotretinoin therapy for acne. Arch Ophthalmol 104:831-837. CrossRef Medline

Wong KY (2012) A retinal ganglion cell that can signal irradiance continuously for $10 \mathrm{~h}$. J Neurosci 32:11478-11485. CrossRef Medline

Wong KY, Dunn FA, Berson DM (2005) Photoreceptor adaptation in intrinsically photosensitive retinal ganglion cells. Neuron 48:1001-1010. CrossRef Medline

Xue Y, Shen SQ, Jui J, Rupp AC, Byrne LC, Hattar S, Flannery JG, Corbo JC, Kefalov VJ (2015) CRALBP supports the mammalian retinal visual cycle and cone vision. J Clin Invest 125:727-738. CrossRef Medline 